\title{
Purity Estimation with Separable Measurements
}

\author{
E. Bagan, ${ }^{1}$ M. A. Ballester, ${ }^{2}$ R. Muñoz-Tapia, ${ }^{1}$ and O. Romero-Isart ${ }^{1}$ \\ ${ }^{1}$ Grup de Física Teòrica \& IFAE, Facultat de Ciències, Edifici Cn, Universitat Autònoma de Barcelona, \\ 08193 Bellaterra (Barcelona), Spain \\ ${ }^{2}$ Department of Mathematics, University of Utrecht, Box 80010, 3508 TA Utrecht, The Netherlands
}

(Received 17 June 2005; published 9 September 2005)

\begin{abstract}
Given a large number $N$ of copies of a qubit state of which we wish to estimate its purity, we prove that separable-measurement protocols can be as efficient as the optimal joint-measurement one if classical communication is used. This shows that the optimal estimation of the entanglement of a two-qubit state can also be achieved asymptotically with fully separable measurements. Thus, quantum memories provide no advantage in this situation. The relationship between our global Bayesian approach and the quantum Cramér-Rao bound is discussed.
\end{abstract}

DOI: 10.1103/PhysRevLett.95.110504

PACS numbers: 03.67.Hk, 03.65.Ta

The ultimate goal of quantum state estimation is to determine the value of the parameters that fully characterize a given unknown quantum state. However, in practical applications, a partial characterization is often all one needs. Thus, e.g., knowing the purity of a qubit state or the degree of entanglement of a bipartite state may be sufficient to determine whether it can perform some particular task [1] — see Ref. [2] for recent experimental progress on estimating the degree of polarization (the purity) of light beams. This Letter concerns this type of situation.

To be more specific, assume we are given $N$ identical copies of an unknown qubit mixed state $\rho(\vec{r})$, so that the state of the total system is $\rho^{N}(\vec{r}) \equiv[\rho(\vec{r})]^{\otimes N}$. The set of all such density matrices $\{\rho(\vec{r})\}$ can be mapped into the Bloch sphere $\mathcal{B}=\{\vec{r}: r \equiv|\vec{r}| \leq 1\}$ through the relation $\rho(\vec{r})=$ $(\mathbb{1}+\vec{r} \cdot \vec{\sigma}) / 2$, where $\vec{\sigma}=\left(\sigma_{x}, \sigma_{y}, \sigma_{z}\right)$ is a vector made out of the three standard Pauli matrices. Our aim is to estimate the purity, $r$, as accurately as possible by performing suitable measurements on the $N$ copies, i.e., on $\rho^{N}(\vec{r})$. This problem can also be viewed as the parameter estimation of a depolarizing channel [3] when it is fed with $N$ identical states.

Estimation protocols are broadly divided into two classes depending on the type of measurements they use: joint and separable. The former treats the system of $N$ qubits as a whole, allowing for the most general measurements, and leads to the most accurate estimates or, equivalently, to the largest fidelity (properly defined below). The latter treats each copy separately, but classical communication can be used in the measurement process. This class is particularly important because it is feasible with current technology and it offers an economy of resources. In this Letter we show that for a sufficiently large $N$, separable-measurement protocols for purity estimation can attain the optimal joint-measurement fidelity bound. The power of separable-measurement protocols in achieving optimal performance has also been demonstrated in other contexts [4].
It has been shown [5] that given $N$ copies of a bipartite qubit pure state, $|\Psi\rangle_{A B}$, the optimal protocol for measuring its entanglement consists in estimating the purity of $\rho(\vec{r}) \equiv$ $\operatorname{tr}_{B}\left(|\Psi\rangle_{A B}\langle\Psi|\right)$, where $\operatorname{tr}_{B}$ is the partial trace over the Hilbert space of party $B$ (see [6] for related work on bipartite mixed states). We thus show that for large $N$ this entanglement can be optimally estimated by performing just separable measurements on one party (party $A$ in this discussion) of each of the $N$ copies of $|\Psi\rangle_{A B}$.

In this Letter, special attention is paid to the asymptotic regime, when $N$ is large. There are several reasons for this. First, in this limit, formulas greatly simplify and usually reveal important features of the estimation protocol. Second, the asymptotic theory of quantum statistical inference, which has become in recent years a very active field in mathematical statistics [7], deals with problems such as the one at hand. Our results give support to some quantum statistical methods for which only heuristic proofs exist; e.g., the applicability of the integrated quantum CramérRao bound in the Bayesian approach [8].

In the first part of this Letter we state some important results concerning the optimal joint estimation protocols and give the corresponding fidelity bounds. In addition to the general case of states in $\mathcal{B}$, which was partially addressed in [5], we also consider the situation when the unknown state is constrained to lie on the equatorial plane $\mathcal{E}$ of the Bloch sphere $\mathcal{B}$. In the second part, we discuss separable-measurement protocols, we prove that they saturate the joint-measurement bound asymptotically, and we state our conclusions.

Mathematically, the problem of estimating the purity of $\rho(\vec{r})$ can be formulated within the Bayesian framework as follows (see [9] for a large deviations approach, which is meaningful only in the asymptotic regime). Let $\mathcal{R}_{\mathcal{O}}=$ $\left\{R_{\chi}\right\}$ be the set of estimates of $r$, each of them based on a particular outcome $\chi$ of some generalized measurement $\mathcal{O}$ over $\rho^{N}(\vec{r})$. Such measurement is characterized by a positive operator valued measure (POVM), namely, by a set of positive operators $\mathcal{O}=\left\{O_{\chi}\right\}$ that satisfy $\sum_{\chi} O_{\chi}=\mathbb{1}$. A 
separable measurement is a particularly interesting instance of a POVM for which each $O_{\chi}$ is a tensor product of $N$ individual operators (usually projectors) each one of them acting on $\rho(\vec{r})$.

Next, a figure of merit, $f\left(r, R_{\chi}\right)$, is introduced as a quantitative way of expressing the quality of the purity estimation. Throughout this Letter we use

$$
f\left(r, R_{\chi}\right)=r R_{\chi}+\sqrt{1-r^{2}} \sqrt{1-R_{\chi}^{2}},
$$

which we call fidelity for short. Its values are in the range $[0,1]$, where unity corresponds to perfect determination. This fidelity has a natural interpretation: in Uhlmann's geometric representation of the set of density matrices as the hemisphere $(1 / 2) \mathbb{S}^{3} \subset \mathbb{R}^{4}$, the function $D\left(r, R_{\chi}\right)=$ $(1 / 2) \arccos f\left(r, R_{\chi}\right)$ is the geodesic (Bures) distance [10] between two sets (two parallel two-dimensional spheres) characterized by the purities $r$ and $R_{\chi}$, respectively.

The optimal protocol is obtained by maximizing $F\left(\mathcal{O}, \mathcal{R}_{\mathcal{O}}\right)=\sum_{\chi} \int d \rho f\left(r, R_{\chi}\right) \operatorname{tr}\left[\rho^{N}(\vec{r}) O_{\chi}\right]$, where $d \rho$ is the prior probability distribution of $\rho(\vec{r})$, and we identify the trace as the probability of obtaining the outcome $\chi$ given that the state we measure upon is $\rho^{N}(\vec{r})$. Thus, $F$ is the average fidelity. The maximization is over the estimator (guessed purity) $\mathcal{R}_{\mathcal{O}}$ and the POVM $\mathcal{O}$,

$$
F^{\max } \equiv \max _{\mathcal{O}}\left\{\max _{\mathcal{R}_{\mathcal{O}}} F\left(\mathcal{O}, \mathcal{R}_{\mathcal{O}}\right)\right\}
$$

In this formulation, we need to provide a prior probability distribution (prior for short) $d \rho$, which encodes our initial knowledge about $\rho(\vec{r})$. Here we assume to be completely ignorant of both $\vec{n} \equiv \vec{r} / r$ and $r$. Our lack of knowledge about the former is properly represented with the choice $d \rho \propto d \Omega$ (solid angle element), which states that $\grave{a}$ priori $\vec{n}$ is isotropically distributed on $\mathcal{B}$. Therefore, we write

$$
d \rho=\frac{d \Omega}{4 \pi} w(r) d r ; \quad \int_{0}^{1} d r w(r)=1 .
$$

While there is wide agreement on this respect, the $r$ dependence of the prior is controversial, and so far we will not stick to any particular choice. Nevertheless, it is worth keeping in mind that the hard sphere prior $w(r)=3 r^{2}$ shows up in the context of entanglement estimation [11], whereas the Bures prior $w(r)=(4 / \pi) r^{2}\left(1-r^{2}\right)^{-1 / 2}$ is most natural in connection with distinguishability of density matrices [12-14].

Rather than computing (2), we here present the main results (details can be found in [15]). (i) The optimal POVM is defined by the set of operators $\left\{\mathbb{1}_{j \alpha}=\right.$ $\left.\sum_{m}|j m ; \alpha\rangle\langle j m ; \alpha|\right\}$. Each $\mathbb{1}_{j \alpha}$ projects over the invariant subspace corresponding to an irreducible representation $\mathbf{j}$ of $S U(2)$ - the group of unitary transformations $U$ that acts naturally over $\mathcal{B}$ as $\rho(\vec{r}) \rightarrow U \rho(\vec{r}) U^{\dagger}$. Here the index $\alpha$ $\left(\alpha=1,2, \ldots, n_{j}\right)$ labels the different $n_{j}$ occurrences of $\mathbf{j}$.
All these $n_{j}$ equivalent representations $\mathbf{j}$ give an identical contribution to $F$. This result should not come as a surprise. The optimal purity estimate, and thus the fidelity, should depend only on invariant quantities (i.e., $j$ and $\alpha$ ), as the purity itself is rotationally invariant, and so is our choice for the prior. (ii) The optimal purity estimator can be written as $R_{j}=A_{j}\left(A_{j}^{2}+B_{j}^{2}\right)^{-1 / 2}$, where

$$
\left(A_{j}, B_{j}\right)=\int d r w(r)\left(r, \sqrt{1-r^{2}}\right) \sum_{m=-j}^{j} p_{r}^{N / 2-m} q_{r}^{N / 2+m},
$$

and $p_{r}=(1-r) / 2, q_{r}=1-p_{r}$. We can easily identify the sum in (4) as the probability $\operatorname{tr}\left[\rho^{N}(\vec{r}) \mathbb{1}_{j \alpha}\right]$. (iii) The maximum fidelity is given by

$$
F^{\max }=\left(\begin{array}{c}
N \\
\frac{N}{2}-j
\end{array}\right) \frac{2 j+1}{\frac{N}{2}+j+1} \sum_{j} \sqrt{A_{j}^{2}+B_{j}^{2}},
$$

where the coefficient in front of the sum is $n_{j}[8,16]$.

For large $N$, this can be computed to be [8]

$$
F^{\max }=1-\frac{1}{2 N}+o\left(N^{-1}\right) .
$$

One can also check that at leading order $R_{j}=2 j / N+\ldots$, as one would intuitively expect. These asymptotic results hold for any prior $w(r)$.

It is also interesting to analyze the case where $\vec{r}$ is known to lie on the equatorial plane $\mathcal{E}$. With this information, the prior probability distribution becomes $d \rho=$ $(d \phi / 2 \pi) w(r) d r$, where $\phi$ is the polar angle of the spherical coordinates. The group of unitary transformations on $\mathcal{E}$ is now a $U(1)$ subgroup of $S U(2)$ and, hence, the optimal POVM is given by the corresponding one-dimensional projectors over the $U(1)$-invariant subspaces, $\left\{\mathbb{1}_{j \alpha m} \equiv\right.$ $|j m ; \alpha\rangle\langle j m ; \alpha|\}$. With this, one can work out the maximum fidelity. It turns out that asymptotically $F^{\max }$ is also given by (6) and the optimal guess is $R_{j m}=2 j / N+\ldots$ (independently of $m$ ). The same conclusions also hold in the one-dimensional case of states known to lie on a diameter of $\mathcal{B}$. Therefore, we see that the information about $\vec{n}$ becomes irrelevant in the asymptotic limit.

A word regarding quantum statistical inference is in order here. It is often argued that the quantum CramérRao bound [17] can be integrated to provide an attainable asymptotic lower bound for some averaged figures of merit, such as the fidelity (1). Ours is a so-called one parameter problem for which the quantum Cramér-Rao bound takes the simple form $\operatorname{Var} R \geq H^{-1}(\vec{r}) / N$, where $\operatorname{Var} R \equiv\left\langle\left(R_{\chi}-\left\langle R_{\chi}\right\rangle\right)^{2}\right\rangle$ is the variance of the estimator $R_{\chi}$, the average is over the outcomes $\chi$ of a measurement, $H(\vec{r})$ is the quantum information matrix [17], and $R_{\chi}$ is assumed to be unbiased: $\left\langle R_{\chi}\right\rangle=r$. In our case $H(\vec{r})=\left(1-r^{2}\right)^{-1}$, and the bound is attainable. This provides in turn an attainable asymptotic upper bound for the fidelity (1), since $\left\langle f\left(r, R_{\chi}\right)\right\rangle \approx 1-\frac{1}{2} H(\vec{r}) \operatorname{Var} R+\ldots$ Assuming one can in- 
tegrate this relation over the whole of $\mathcal{B}$ [including the region $r \approx 1$, where $H(\vec{r})$ is singular], with a weight function given by the prior (3), one easily obtains Eq. (6). Unfortunately, there are only heuristic arguments supporting this assumption, but so far no rigorous proof exists in the literature.

We now abandon the joint protocols to dwell on separable-measurement strategies for the rest of the Letter. Here we focus on the asymptotic regime, but some brief comments concerning small $N$ can be found in the conclusions.

In previous work [18], some of the authors showed that the maximum fidelity one can achieve in estimating both $r$ and $\vec{n}$ (full estimation of a qubit mixed state) assuming the Bures prior and using tomography behaves as $F_{\text {full }}^{\max }=1-$ $\xi N^{-3 / 4}+o\left(N^{-3 / 4}\right)$, where $\xi$ is a positive constant. The same behavior one should expect for our fidelity $F^{\max }$, since the effect of the purity estimation is dominant in $F_{\text {full }}^{\max }$. This strange power law, somehow unexpected on statistical grounds, is caused by the behavior of $w(r)$ in a small region $r \approx 1$. Indeed, it is not difficult to convince oneself that if $w(r) \propto\left(1-r^{2}\right)^{-\lambda} \approx 2(1-r)^{-\lambda}$ for $r \approx 1$, one should have $1-F^{\max } \propto N^{\lambda / 2-1}+\ldots$, for $0<\lambda<1$ (for $\lambda=0$, hard sphere prior, one should have logarithmic corrections). This differs drastically from (6) which, as stated above, holds for any such values of $\lambda$. Would classical communication be enough to restore the right power law $N^{-1}$ for $1-F^{\max }$ and, moreover, saturate the bound of the optimal joint-measurement protocol?

On quantum statistical grounds, one should expect a positive answer to this question since the quantum Cramér-Rao bound is attained by a separable protocol consisting in performing the (von Neumann) measurements $\mathcal{M}=\{(\mathbb{1} \pm \vec{n} \cdot \sigma) / 2\}$ on each copy. Note, however, that $\mathcal{M}$ depends on $\vec{n}$, which is, of course, unknown à priori. This protocol can make sense only if we are ready to spend a fraction of the $N$ copies of $\rho(\vec{r})$ to obtain an estimate of $\vec{n}$, use this classical information to design $\mathcal{M}$, and, finally, perform this adapted measurement on the remaining copies. This protocol was successfully applied to pure states by Gill and Massar in [19]. We extend it to a purity estimation below.

Let us consider a family of priors of the form

$$
w(r)=\frac{4}{\sqrt{\pi}} \frac{\Gamma(5 / 2-\lambda)}{\Gamma(1-\lambda)} r^{2}\left(1-r^{2}\right)^{-\lambda},
$$

which includes both the Bures $(\lambda=1 / 2)$ and the hard sphere $(\lambda=0)$ metrics. Despite this particular $r$ dependence, the final results apply to any prior whose behavior near $r=1$ is given by (7).

We now proceed à la Gill-Massar [19] and consider the following one-step adaptive protocol: we take a fraction $N^{\alpha} \equiv N_{0}(0<\alpha<1)$ of the $N$ copies of $\rho(\vec{r})$ and use them to estimate $\vec{n}$. Tomography along the three orthogonal axes $x, y$, and $z$, together with a very elementary estimation based on the relative frequencies of the outcomes [20], enables us to estimate $\vec{n}$ with an accuracy given by

$$
\frac{\left\langle\Theta_{r}^{2}\right\rangle}{2} \approx 1-\left\langle\cos \Theta_{r}\right\rangle=\frac{3}{N_{0}}\left(\frac{1}{r^{2}}-\frac{1}{5}\right)+o\left(N_{0}^{-1}\right),
$$

where $\Theta_{r}$ is the angle between $\vec{n}$ and its estimate. Here and below $\langle\cdots\rangle$ is not only the average over the outcomes of these tomography measurements, but also contains an integration over the prior angular distribution $d \Omega /(4 \pi)$ for fixed $r$.

In a second step, we measure the projection of $\vec{\sigma}$ along the estimated $\vec{n}$ obtained in the previous step. We perform this von Neumann measurement on each of the remaining $N-N_{0} \equiv N_{1}$ copies of the state $\rho(\vec{r})$. We estimate the purity to be $R=2 N_{+} / N_{1}-1$, where $N_{ \pm} / N_{1}$ is the relative frequency of \pm 1 outcomes, and we drop the $N_{+}$ dependence of $R$ to simplify the notation.

Obviously, as a random variable and for large $N_{1}, R$ is normally distributed as $R \sim N\left(r c_{r}, \sqrt{1-r^{2} c_{r}^{2}} / \sqrt{N_{1}}\right)$, where $c_{r}=\cos \Theta_{r}$. Hence, for large $N_{0}$ and $N_{1}$ it makes sense to expand $f(r, R)$, Eq. (1), around $R=r c_{r}$, and thereafter, because of (8), expand the resulting expression around $c_{r}=1$. We obtain

$$
F(r)=1-\frac{1}{2 N_{1}}+\frac{r^{2}}{1-r^{2}}\left(\frac{\left\langle\Theta_{r}^{2}\right\rangle}{4 N_{1}}-\frac{\left\langle\Theta_{r}^{4}\right\rangle}{8}\right)+\ldots,
$$

where $F(r)$ is the average fidelity for fixed $r$, i.e., $\int d r w(r) F(r)=F$. In view of $(8),\left\langle\Theta_{r}^{4}\right\rangle \sim N_{0}^{-2}=N^{-2 \alpha}$. Hence, the two terms in parentheses in (9) can be dropped only if $\alpha>1 / 2$. Provided $w(r)$ vanishes as in (7) with $\lambda<$ 0 , we can integrate $r$ in (9) over the unit interval to obtain

$$
F=1-\frac{1}{2 N\left(1-N^{\alpha-1}\right)}+o\left(N^{-1}\right),
$$

and we conclude that this protocol attains asymptotically the joint-measurement bound (6).

However, most of the physically interesting priors [11,14], $w(r)$, not only do not vanish as $r \rightarrow 1$, but often diverge like (7) with $0<\lambda<1$. In this case (9) cannot be integrated, as the last term does not lead to a convergent integral. This signals that the series expansion around $c_{r}=1$ leading to (9) is not legitimated in the whole of $\mathcal{B}$.

To fix the problem, we split $\mathcal{B}$ in two regions. A sphere of radius $1-\epsilon, \epsilon>0$, which we call $\mathcal{B}^{\mathrm{I}}$, and a spherical sheet of thickness $\epsilon$ : $\mathcal{B}^{\mathrm{II}}=\{\vec{r}: 1-\epsilon<r \leq 1\}$. The fidelity can thus be written as the sum of the corresponding two contributions: $F=F^{\mathrm{I}}+F^{\mathrm{II}}$. While $F^{\mathrm{I}}$ can be obtained by simply integrating (9) over $\mathcal{B}^{\mathrm{I}}$, where this expansion is valid, some care must be taken in the region $\mathcal{B}^{\text {II }}$. There, we proceed as follows.

We compute the fidelity as if all the states in $\mathcal{B}^{\mathrm{II}}$ had the lowest possible purity $(r=1-\epsilon)$ when the first-step tomography was performed. This leads to a lower bound for $F^{\mathrm{II}}$, because the lower the purity of a state the less accurately $\vec{n}$ can be determined [see Eq. (8)], and hence, the 
worse its purity can be estimated in the second step. The trick, which amounts to replacing $c_{r}$ by $c_{1-\epsilon}$, enables us to integrate $r$ prior to performing the average $\langle\cdots\rangle$. A straightforward calculation leads to

$$
F \gtrsim 1-\frac{1}{2 N_{1}}-2^{\lambda-2} k_{\lambda}\left\langle\Theta_{1-\epsilon}^{2}\right\rangle^{2-\lambda}+\ldots,
$$

$0<\lambda<1, \quad$ where $\quad k_{\lambda}=2^{2-\lambda} \Gamma\left(\frac{5}{2}-\lambda\right) \Gamma\left(\frac{3}{2}-\lambda\right) \Gamma(\lambda-$ 2) $/[\pi \Gamma(1-\lambda)]$. Now, we can safely take the limit $\epsilon \rightarrow$ 0 . We see that by choosing $\max \{1 / 2,1 /(2-\lambda)\}<\alpha<1$ we ensure that the joint-measurement bound (6) is attained. It is worth emphasizing that the last term in (11), which is completely missing in (10), is actually the dominant contribution if $\alpha<1 /(2-\lambda)$. For $\lambda=0$ we have $F^{\text {hard }} \geq$ $1-(1 / 2) N_{1}^{-1}-(3 / 8) N_{1}^{-1}\left\langle\Theta_{1}^{2}\right\rangle \log \left\langle\Theta_{1}^{2}\right\rangle+\ldots, \quad$ and $\quad$ we again conclude that the protocol presented here attains the joint-measurement bound.

At this point one may wonder if the conclusions above depend upon our particular choice of figure of merit. To get a grasp on this, it is worth using again the standard pointwise approach to quantum statistics. There, one is interested in the mean square error MSER $=\left\langle(R-r)^{2}\right\rangle$ for fixed $r$, where now the average $\langle\cdots\rangle$ is over the outcomes of all measurements for a fixed $\vec{r}$. One can write MSE $R=$ $\operatorname{Var} R+(\langle R\rangle-r)^{2}$, where the second term is the bias. Using the same one-step adaptive protocol described above, we get that the mean square error after step two is MSER $=\left[N_{1} H(r)\right]^{-1}+r^{2}\left\langle\Theta_{r}^{4}\right\rangle / 4+\ldots$ As above, the last term can be dropped if $\alpha>1 / 2$, and $\operatorname{MSE} R=$ $[N H(r)]^{-1}+o\left[N^{-1}\right]$, saturating the quantum CramérRao bound. This protocol is, therefore, also asymptotically optimal in the present context.

In summary, though the absolute bounds for the average fidelity involve joint measurements, these bounds can be obtained asymptotically with separable measurements. This requires classical communication among the sequential von Neumann measurements performed on each of the $N$ individual copies of the state. This result, which has been speculated on quantum statistical grounds, is here proved for the first time by a direct calculation. Since the purity is an optimal measure of the entanglement of a pure bipartite qubit state, we also obtain the additional result that this entanglement can be optimally estimated with separable measurements on just one of the parties.

For finite (but otherwise arbitrary) $N$, finding the optimal separable-measurement protocol is an open problem. Interestingly enough, a "greedy" protocol designed to be optimal at each measurement step [4] leads to an unacceptably poor estimation. Notice that in the one-step adaptive protocol described above, part of the copies were spent ("wasted" from a greedy point of view) in estimating $\vec{n}$. We have seen that this strategy pays in the long run. However, the greedy strategy optimizes measurements in the short run, which translates into measuring $\vec{\sigma}$ along the same arbitrarily fixed axis. This yields a low value for the fidelity, which does not even converge to unity in the strict limit $N \rightarrow \infty$. This counterintuitive behavior also appears in other contexts as, e.g., economics, biology, or social sciences (see, e.g., [21]).

We thank Antonio Acín, Richard Gill, and Juanma Parrondo for useful discussions. This work is supported by the Spanish Ministry of Science and Technology Project No. BFM2002-02588, CIRIT Project No. SGR-00185, Netherlands Organization for Scientific Research NWO, the European Community projects QUPRODIS Contract No. IST-2001-38877 and RESQ Contract No. IST-200137559 .

[1] A. G. White et al., Phys. Rev. Lett. 83, 3103 (1999).

[2] M. Legre, M. Wegmueller, and N. Gisin, Phys. Rev. Lett. 91, 167902 (2003).

[3] M. Sasaki, M. Ban, and S. M. Barnett, Phys. Rev. A 66, 022308 (2002); A. Fujiwara, Phys. Rev. A 70, 012317 (2004).

[4] D. G. Fisher, S. H. Kienle, and M. Freyberger, Phys. Rev. A 61, 032306 (2000); Th. Hannemann et al., Phys. Rev. A 65, 050303 (2002); A. Acin et al., ibid. 71, 032338 (2005); E. Bagan, A. Monras, and R. Munoz-Tapia, ibid. 71, 062318 (2005).

[5] A. Acin, R. Tarrach, and G. Vidal, Phys. Rev. A 61, 062307 (2000).

[6] P. Horodecki, Phys. Rev. Lett. 90, 167901 (2003).

[7] Asymptotic Theory Of Quantum Statistical Inference: Selected Papers, edited by Masahito Hayashi (World Scientific, Singapore, 2005).

[8] E. Bagan et al. (to be published).

[9] M. Keyl and R. F. Werner, Phys. Rev. A 64, 52311 (2001).

[10] H. J. Sommers and K. Zyczkowski, J. Phys. A 36, 10083 (2003).

[11] K. Zyczkowski and H. J. Sommers, J. Phys. A 34, 7111 (2001); H. J. Sommers and K. Zyczkowski, ibid. 37, 8457 (2004).

[12] C. A. Fuchs, Ph.D. thesis, dissertation, University of New Mexico, 1995 (quant-ph/9601020).

[13] M. Hübner, Phys. Lett. A 163, 239 (1992); R. Jozsa, J. Mod. Opt. 41, 2315 (1994).

[14] D. Petz and C. Sudar, J. Math. Phys. (N.Y.) 37, 2662 (1996).

[15] E. Bagan et al., quant-ph/0505083.

[16] J. I. Cirac, A. K. Ekert, and C. Macchiavello, Phys. Rev. Lett. 82, 4344 (1999).

[17] A. Holevo, Probabilistic and Statistical Aspects of Quantum Theory (North-Holland Publishing, Amsterdam, 1982).

[18] E. Bagan, M. Baig, R. Munoz-Tapia, and A. Rodriguez, Phys. Rev. A 69, 010304 (2004).

[19] R. D. Gill and S. Massar, Phys. Rev. A 61, 042312 (2000).

[20] E. Bagan, M. Baig, and R. Munoz-Tapia, Phys. Rev. Lett. 89, 277904 (2002); E. Bagan, A. Monras, and R. MunozTapia, Phys. Rev. A 71, 062318 (2005).

[21] L. Dinis and J. M. R. Parrondo, Europhys. Lett. 63, 319 (2003). 